Urol. Prax. 2021 · 23:40-50

https://doi.org/10.1007/s41973-021-00143-0

Angenommen: 18. Mai 2021

Online publiziert: 29. Juni 2021

(c) Der/die Autor(en) 2021

\section{Allgemeines}

Ungewollte Kinderlosigkeit ist ein ernst zu nehmendes Problem der Gesellschaft. Für viele Paare stellt der Wunsch nach einem Kind einen zentralen Lebensinhalt dar. So ist es nicht verwunderlich, dass das Vorliegen einer Sterilität sowohl $\mathrm{zu}$ einer reduzierten Lebensqualität und vermindertem Selbstwertgefühl als auch $\mathrm{zu}$ persönlicher Verzweiflung und sogar sozialer Isolation führen kann.

Gemäss der „World Health Organisation" (WHO) liegt eine Sterilität vor, wenn trotz regelmässigem und ungeschütztem Geschlechtsverkehr nach 1 Jahr noch keine Schwangerschaft eingetreten ist [1].

Die Prävalenz variiert je nach Land sowie der zugrunde gelegten Definition der Sterilität. So wird in einer systematischen Analyse von 190 Ländern eine Prävalenz zwischen $1 \%$ und $24 \%$ angegeben. In der westlichen Welt wird geschätzt, dass ca. jedes 7. Paar ungewollt kinderlos ist [2].

\section{Gesetzliche Grundlage in der Schweiz}

Gesetzliche Grundlage der assistierten reproduktionsmedizinischen Techniken (ART) sind der schweizerische Bundesverfassungsartikel BV Art. 24 $4^{\text {novies }}$, seit 2000 BV Art. 119, sowie das schweizerische Fortpflanzungsmedizingesetz (FMedG), welches am 01.01.2001 in Kraft trat und dessen revidierte Form seit 01.09.2017 gültig ist. Dieses Gesetz legt fest, unter welchen Voraussetzungen die Verfahren der medizinisch unterstützten Fortpflanzung beim Menschen angewendet werden dürfen. Nur wenn die Unfruchtbarkeit eines Paares überwunden werden soll und die anderen Behandlungsmethoden versagen, aus-

\title{
Ruth Stiller
}

Universitäres Kinderwunschzentrum, Klinik für Reproduktions-Endokrinologie, Universitätsspital Zürich, Zürich, Schweiz

\section{Assistierte Fertilisation}

sichtslos sind oder die Gefahr, dass eine schwere, unheilbare Krankheit auf die Nachkommen übertragen wird, anders nicht abgewendet werden kann, darf ein assistiertes Fortpflanzungsverfahren durchgeführt werden (Art. 5). Eine Bewilligung des Kantons benötigt, wer Fortpflanzungsverfahren anwendet, Keimzellen, imprägnierte Eizellen oder Embryonen in vitro zur Kryokonservierung entgegennimmt oder gespendete Samenzellen vermittelt, ohne selber Fortpflanzungsverfahren anzuwenden. Für die Insemination mit Samenzellen des Partners ist keine Bewilligung erforderlich (Art. 8). Jährlich muss ein Bericht über die Zahl und Art der Behandlungen, die Indikation, die Verwendung gespendeter Samenzellen, die Zahl der Schwangerschaften und deren Ausgang, die Konservierung und Verwendung von Keimzellen und imprägnierten Eizellen und die Anzahl überzähliger Embryonen der Bewilligungsbehörde zugestellt werden. Diese übermittelt die Daten zur Auswertung und Veröffentlichung (Art. 11) dem Bundesamt für Statistik. Ferner erfolgen regelmässige Kontrollen des reproduktionsmedizinischen Zentrums durch die Bewilligungsbehörde (Art. 12).

Im Gegensatz zu dem vorab gültigen Fortpflanzungsmedizingesetz ist es nun auch möglich, die Präimplantationsdiagnostik an Embryonen vorzunehmen. Die Untersuchung des Erbguts darf aber nur „zur Erkennung chromosomaler Eigenschaften, die die Entwicklungsfähigkeit des zu zeugenden Embryos beeinträchtigen können, oder wenn die Gefahr, dass die Veranlagung für eine schwere Krankheit übertragen wird, anders nicht abgewendet werden kann“ vorgenommen werden (Art. 5). Im Zuge der Möglichkeit, die Präimplantationsdi- agnostik durchführen zu können, dürfen nun auch Embryonen kryokonserviert werden, was bisher untersagt war. Vor dem 01.09.2017 war es nur erlaubt, befruchtete Oozyten zu kryokonservieren, Embryonen mussten alle transferiert werden.

\section{FIVNAT-Register}

Die damalige Schweizerische Gesellschaft für Fertilität, Sterilität und Familienplanung gründete 1992 die Arbeitsgruppe FIVNAT-CH. Diese implementierte ein Register, welches anonymisierte und detaillierte Angaben zu den durchgeführten In-vitro-Fertilisations(IVF) und intrazytoplasmatischen Spermieninjektionsbehandlungen (ICSI-Behandlungen) der Schweiz in den jeweils angeschlossenen Zentren sammelt und statistisch auswertet. Seit 1997 werden jährlich externe Qualitätskontrollen in den teilnehmenden Zentren durchgeführt, um die Korrektheit der Angaben $\mathrm{zu}$ überprüfen. Jedes Zentrum erhält einmal jährlich von der FIVNAT-CH die Daten seiner eigenen Behandlungen, welche mit den Daten der restlichen Zentren der Schweiz verglichen werden. Die Mitgliedschaft ist freiwillig, und deren Finanzierung wird durch die Zentren selber gesichert. Momentan sind alle Schweizer IVF-Zentren Mitglied von FIVNAT-CH.

\section{Vorabklärungen vor einer Sterilitätstherapie}

Bevor bei einem Paar eine Sterilitätstherapie durchgeführt wird, muss zunächst die Ursache der Sterilität evaluiert werden. Hierzu sollten der endokrine, der mechanische sowie der männliche Faktor abgeklärt werden. Ferner werden wich- 


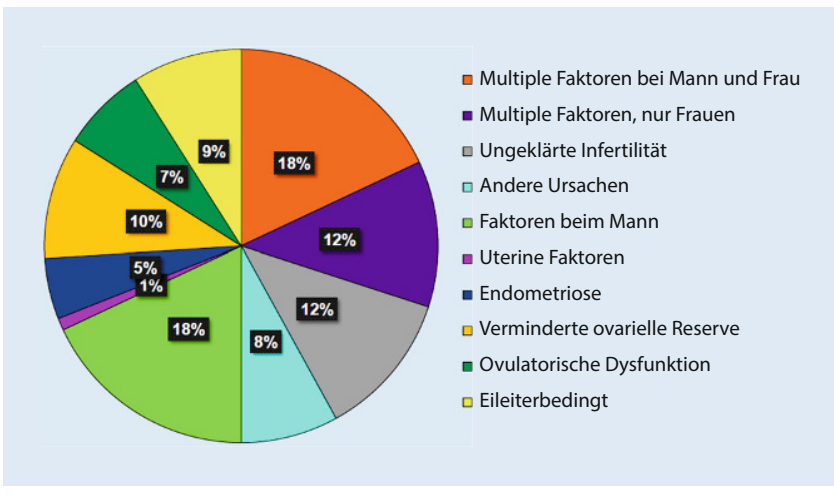

tätstherapie gewählt werden. Im Folgenden wird die Vorgehensweise der IVF und ICSI vorgestellt.

\section{In-Vitro-Fertilisations-/ Intrazytoplasmatische Spermieninjektions- Behandlungen}

Abb. $1<$ Häufigkeitsverteilung der Sterilitätsursachen (Basierend auf [3]) tige Infektionen wie HIV, Hepatitis B und C, Chlamydia trachomatis sowie die Rubeolen- und Varizellenimmunität untersucht. Wichtig ist, dass immer beide Partner abgeklärt werden, da bei fast $20 \%$ aller Paare sowohl beim Mann als auch der Frau eine Sterilitätsursache gefunden werden kann (• Abb. 1).

Unter dem endokrinen Faktor werden u. a. ovulatorische Dysfunktionen sowie die ovarielle Reserve verstanden.

Als Biomarker für die persönliche ovarielle Reserve wird bereits seit den 1990er Jahren der basale FSH-Wert verwendet. Die Blutentnahme sollte frühzyklisch, optimalerweise zwischen dem 2. und 4. Zyklustag, erfolgen. Es ist bekannt, dass ein erhöhter FSH-Wert einen Hinweis auf eine verminderte ovarielle Reserve darstellt [4] und als guter Prädiktor für den fehlenden Schwangerschaftseintritt bei der Sterilitätstherapie gilt [5]. Hinsichtlich des sinnvollen Cutoff-Wertes gibt es keine allgemeingültige Definition. Je nach Studie wird dieser Grenzwert mit 8, 10, 15 oder 20 IU/1 angegeben [6].

Mittlerweile tritt immer mehr das Anti-Müller-Hormon (AMH) als Biomarker in den Vordergrund. Es wird in den Granulosazellen der präantralen und kleinen antralen Follikel gebildet und spiegelt damit den follikulären Pool wider. Im Gegensatz zum basalen FSHWert ist die Bestimmung des AMHs zyklusunabhängig. Hinsichtlich seiner Aussagekraft bezüglich einer Lebendgeburt nach IVF-/ICSI-Behandlung kamen die Autoren zweier Metaanalysen zum Schluss, dass AMH, unabhängig vom maternen Alter, eine gewisse Assoziation mit der Vorhersage einer Lebendgeburt bei der Sterilitätstherapie hat $[7,8]$.
Der „antral follicle count“ (AFC) dient der sonographischen Evaluation des follikulären Pools. Hierbei werden die Anzahl antraler Follikel von einem Durchmesser zwischen 2 und $10 \mathrm{~mm}$ in beiden Ovarien gezählt [9]. Er zeigt für die Anzahl gewonnener Oozyten bei einer IVF-/ICSI-Behandlung einen guten prädiktiven Wert, ebenso wie das AMH. Hinsichtlich der Vorhersage eines Schwangerschaftseintritts zeigten Broekmans et al. in ihrem Review eine grosse Spannbreite der Sensitivität mit Werten zwischen 0,07 und 0,60 , wobei die Spezifität mit zwischen 0,33 und 0,98 angegeben wurde [10].

Unter dem mechanischen Faktor werden anatomische Veränderungen verstanden, die das Aufeinandertreffen von Spermien und Oozyte oder die Implantation eines Embryos beeinträchtigen. Als "golden standard“ für diese Abklärung gelten die Chromolaparoskopie und Hysteroskopie. Hierbei können Pathologien direkt dargestellt und, sofern möglich, behoben werden. Dieser operative Eingriff empfiehlt sich insbesondere, wenn aufgrund von Voruntersuchungen ein hochgradiger Verdacht auf eine Pathologie (z. B. sonographisch dargestellte ovarielle Befunde oder anamnestisch ausgeprägte Dysmenorrhö als Hinweis auf eine Endometriose) besteht. Ansonsten können das Cavum uteri und die Tuben radiologisch mittels Hysterosalpingographie (HSG) oder sonographisch durch eine Hydro- bzw. Hysterosalpingokontrastsonographie (HyCoSy) evaluiert werden.

Liegt beim Mann eine Subfertilität vor, sollte diese vom Spezialisten urologischandrologisch abgeklärt werden.

Sofern alle Resultate der Vorabklärungen vorliegen, kann die geeignete Sterili-
Mit der Geburt von Louise Brown, welche am 25.07.1978 in England geboren wurde, trat die assistierte Reproduktionsmedizin in das breite, öffentliche Bewusstsein. Sie war das erste Kind, welches nach einer IVF geboren wurde. Dem Gynäkologen Patrick Steptoe gelang zusammen mit dem Physiologen Robert Edwards dieser Erfolg [11]. Mittlerweile wurden weltweit mehr als 7 Mio. Babys aus IVF-/ICSI-Zyklen geboren. In der Schweiz basieren ca. 2,5\% aller Geburten auf diesen Behandlungen [12].

Um eine erfolgreiche IVF-/ICSI-Behandlung durchzuführen, müssen viele einzelne Schritte optimal ablaufen. Diese werden im Folgenden näher beschrieben.

\section{Ablauf einer IVF-/ICSI- Behandlung}

\section{Ovarielle Stimulation}

Zunächst erfolgt eine kontrollierte ovarielle Hyperstimulation mit Gonadotropinen. Dadurch wird eine Reifung von Oozyten bewirkt, denn nur eine reife Oozyte in der Metaphase II ist befruchtungsfähig. Ziel der Stimulation ist es, zwischen 10 und 15 reife Oozyten zu erhalten, da damit eine optimale Schwangerschaftschance gegeben ist und das Risiko für die Entwicklung eines ovariellen Überstimulationssyndroms als Komplikation nicht erhöht ist [13].

Um einen vorzeitigen LH-Anstieg, welcher zu einer hohen Rate an Stimulationsabbrüchen führen würde, $\mathrm{zu}$ vermeiden, werden zusätzlich Gonadotropin-Releasing-Hormon-Agonisten (GnRHa) oder Gonadotropin-Releasing-Hormon-Antagonisten (GnRH-A) eingesetzt.

Mittels regelmässiger transvaginaler Ultraschalluntersuchung und der Bestimmung von Östradiol und Progesteron im Serum wird die Stimulation 
engmaschig überwacht. Sonographisch werden zum einen die Dicke des Endometriums und zum anderen die Anzahl und Grösse der Follikel bestimmt. Ein optimales Endometrium sollte mehr als $7 \mathrm{~mm}$ Dicke [14] und einen „bilayer“ aufweisen [15]. Hinsichtlich der Follikelgrösse sollten mindestens 3 Follikel einen mittleren Durchmesser von $16-18 \mathrm{~mm}$ haben, da bei dieser Follikelgrösse in den meisten Fällen davon ausgegangen werden kann, dass eine reife Oozyte in der Metaphase II vorhanden ist.

\section{Follikelpunktion}

Sofern die optimalen Follikelgrössen erreicht sind, wird die Endausreifung der Oozyten mittels der Injektion mit hCG initiiert. 34-36 h später erfolgt die Eizellgewinnung mittels transvaginaler Follikelpunktion (siehe - Abb. 2). Bis Mitte der 1980er Jahre erfolgten die Punktionen laparoskopisch, anschliessend sonographisch kontrolliert transabdominal [16]. Mit Hilfe von Vaginalsonden konnte erstmals 1985 die ultraschallkontrollierte transvaginale Follikelpunktion erfolgen [17]. Dieses stellt bis heute das Standardvorgehen dar. Diese Methode ist minimal-invasiv, kann je nach Wunsch der Patientin ohne Narkose durchgeführt werden und erlaubt auch, Follikel in der Tiefe der Ovarien zu punktieren. Komplikationen sind äusserst selten.

\section{IVF/ICSI}

Optimalerweise wird noch während der Punktion die gewonnene Follikelflüssigkeit auf das Vorhandensein einer Oozyte von den Embryologen/Embryologinnen kontrolliert. Diese wird von eventuell vorhandenen Blutkoageln, Luftbläschen und überschüssigen Granulosazellen gereinigt und anschliessend in ein Kulturmedium umgebettet. Eine reife, befruchtungsfähige Oozyte hat den ersten Polkörper ausgestossen und befindet sich in der Metaphase II (• Abb. 3).

Diese reifen Oozyten werden mit den Spermien des Partners zusammengefügt. Vorgängig muss das Sperma aufbereitet werden. Hierzu werden am häufigsten die Methode des „swim-up“ oder die Dichtegradientenfiltration angewendet.

Urol. Prax. 2021 · 23:40-50 https://doi.org/10.1007/s41973-021-00143-0

(c) Der/die Autor(en) 2021

\section{R. Stiller}

\section{Assistierte Fertilisation}

\section{Zusammenfassung}

Ungewollte Kinderlosigkeit ist ein ernst zu nehmendes Problem. Für viele Paare stellt der Wunsch nach einem Kind einen zentralen Lebensinhalt dar. Gemäss der „World Health Organisation" (WHO) liegt eine Sterilität vor, wenn trotz regelmässigem und ungeschütztem Geschlechtsverkehr nach 1 Jahr noch keine Schwangerschaft eingetreten ist. Die Prävalenz variiert je nach Land. Es wird geschätzt, dass in der westlichen Welt ca. jedes 7. Paar betroffen ist. Zahlreiche Gründe können für eine ungewollte Kinderlosigkeit verantwortlich sein. Vor Beginn einer Sterilitätstherapie sollten diese abgeklärt werden, um eine optimale Wahl der Sterilitätstherapie treffen zu können. Die assistiert-reproduktionsmedizinischen Techniken stellen wichtige Therapieoptionen mit guten Erfolgschancen dar. Mit dem Inkrafttreten des revidierten Fortpflanzungsmedizingesetztes der Schweiz ist es nun auch möglich, Verfahren, die früher nur im Ausland erfolgen konnten, im Inland durchzuführen. So kann mittlerweile Paaren mit schweren Erbleiden auch im eigenen Land eine Präimplantationsdiagnostik erfolgreich angeboten werden.

\section{Schlüsselwörter}

Sterilität · In-Vitro-Fertilisation · Intrazytoplasmatische Spermieninjektion . Präimplantationsdiagnostik · Maternes Alter

\section{Fertilisation assistée}

\section{Résumé}

L'absence d'enfant involontaire est un problème qui doit être pris au sérieux. Avoir un enfant est un but de vie essentiel pour de nombreux couples. L'Organisation mondiale de la santé (OMS) définit la stérilité comme l'absence d'une grossesse malgré de réguliers rapports sexuels non protégés pendant une période d'au moins un an. La prévalence varie d'un pays à l'autre. On estime qu'environ un couple sur sept est affecté dans le monde occidental. L'absence d'enfant involontaire peut avoir de nombreuses causes. Avant de commencer un traitement de la stérilité, il faut examiner quelles sont les causes impliquées afin de pouvoir définir le traitement optimal de l'infertilité. Les techniques de procréation médicalement assistée sont des options thérapeutiques importantes qui offrent de bonnes chances de succès. L'entrée en vigueur en Suisse de la loi révisée sur la procréation médicalement assistée permet désormais d'appliquer en Suisse des procédés qui n'étaient auparavant possibles qu'à l'étranger. Ainsi, un diagnostic préimplantatoire peut à présent être proposé avec succès dans leur propre pays aux couples atteints d'anomalies génétiques sévères.

Mots clés

Stérilité · Fécondation in vitro · Injection intracytoplasmique de spermatozoïdes . Diagnostics préimplantatoires · Âge maternel
Zunächst werden das Sperma gewaschen sowie das Seminalplasma entfernt. Beim „swim-up“ müssen anschliessend die Spermien in ein überschichtetes Medium schwimmen, von dem sie dann abpipettiert werden. Somit kann hochmotiles, hochreines Material gewonnen werden. Bei der Dichtegradientenfiltration werden 2 Medien von unterschiedlicher Dichte (z.B. $45 \%$ ige und $90 \%$ ige Lösung) in ein Reagenzglas gegeben, mit der gewaschenen Spermienprobe überschichtet und zentrifugiert. An den Grenzschichten lagern sich auf diese Weise sowohl immotile als auch mal- formierte Spermien, Leukozyten und bakterieller Debris ab. Durch diese Methode kann eine höhere Ausbeute an motilen Spermien auch bei schlechter Spermienqualität gewonnen werden.

Für die Zusammenführung von Spermien und Oozyten stehen 2 Verfahren zur Verfügung.

Bei der IVF werden ca. 100.000 rasch progressive motile Spermien pro Eizelle verwendet. Eizelle und Spermien werden dabei jeweils in $1 \mathrm{ml}$ Kulturmedium in eine Kulturschale gegeben und in den Inkubator gestellt. 
Hier steht eine Anzeige.

\section{曾 Springer}




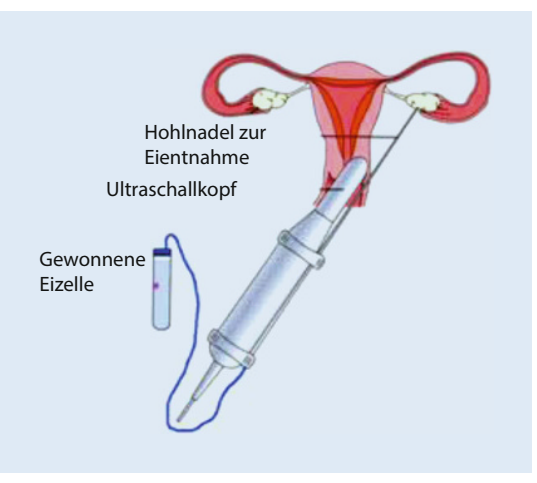

Abb. 2 \ Schematische Darstellung der transvaginalen Follikelpunktion. (Aus: Kinderwunschbroschüre universitäres Kinderwunschzentrum. @ Klinik für Reproduktionsendokrinologie USZ. Diese Abbildung fällt nicht unter die Creative Commons CC BY-Lizenz dieser Publikation.)

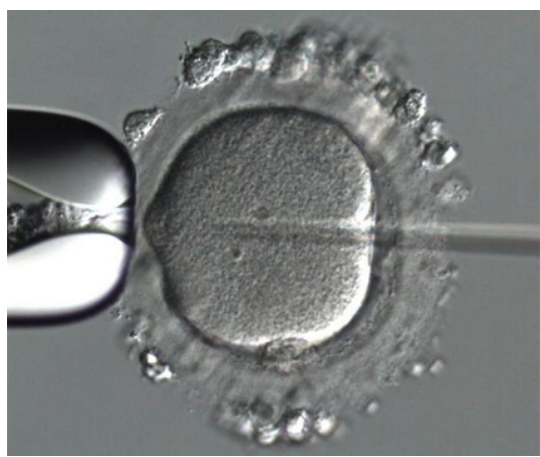

Abb. 5 A Intrazytoplasmatische Spermieninjektion (ICSI). (Mit freundlicher Genehmigung von Dr. Min Xie, universitäres Kinderwunschzentrum USZ. Diese Abbildung fällt nicht unter die Creative Commons CC BY-Lizenz dieser Publikation.)

Hierbei muss es einem Spermium gelingen, in die Oozyte einzudringen und so eine Fertilisation herbeizuführen. In - Abb. 4 ist eine fertilisierte Oozyte mit 2 Vorkernen erkennbar. Sichtbar sind noch zahlreiche Spermien.

Die klassische Indikation für eine IVF ist der beidseitige Tubenverschluss. Weiterhin ist sie bei einer leichten, männlichen Subfertilität sowie nach erfolglosen intrauterinen Inseminationen indiziert. Ebenso kann sie bei einer unerklärten Sterilität angewendet werden, wobei hier das Risiko eines Fertilitätsversagens besteht.

Liegt eine schwere männliche Subfertilität vor, kommt es mittels IVF jedoch mit grosser Wahrscheinlichkeit nicht zu einer Fertilisation, da das Spermium die

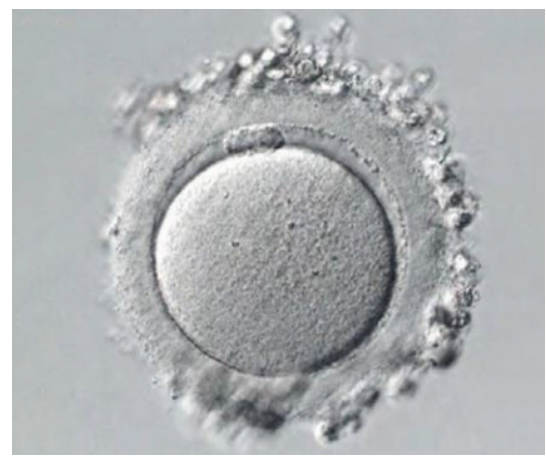

Abb. 3 ॥ Reife, befruchtungsfähige Oozyte. (Mit freundlicher Genehmigung von Dr. Min Xie, universitäres Kinderwunschzentrum USZ. Diese Abbildung fällt nicht unter die Creative Commons CC BY-Lizenz dieser Publikation.)

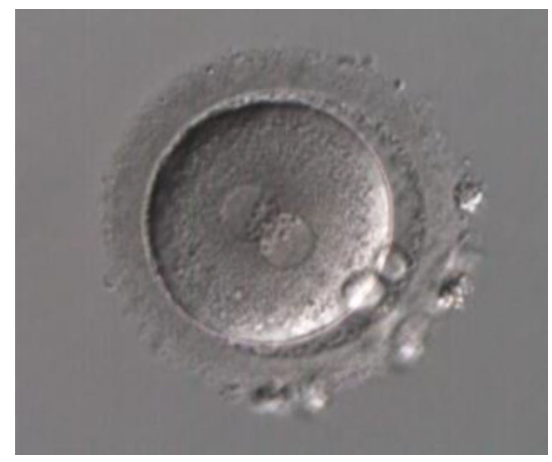

Abb. $6 \Delta$ Normal fertilisierte Oozyte. (Mit freundlicher Genehmigung von Dr. Min Xie, universitäres Kinderwunschzentrum USZ. Diese Abbildung fällt nicht unter die Creative Commons CC BY-Lizenz dieser Publikation.)

Zona pellucida nicht überwinden kann. Verschiedene Ansätze wurden versucht, um diese Problematik zu lösen. So wurde auf Seite der Spermien eine Optimierung mittels z. B. erhöhter Spermienkonzentration oder Zugabe von Koffein und seinen Derivaten zur Verbesserung der Beweglichkeit angewendet. Bei der Oozyte wurde versucht, die Barriere der Zona pellucida mittels ,zona drilling“, partieller Zonadissektion oder einer subzonalen Insemination zu durchbrechen. Die Schwangerschaftsraten waren sehr niedrig $(2,9-16 \%)$ und in über $30 \%$ kam es zu einer Polyspermie [18-20].

Erst durch die Einführung der intrazytoplasmatischen Spermieninjektion ist es möglich geworden, gute Schwangerschaftsraten zu erzielen. Diese Methode

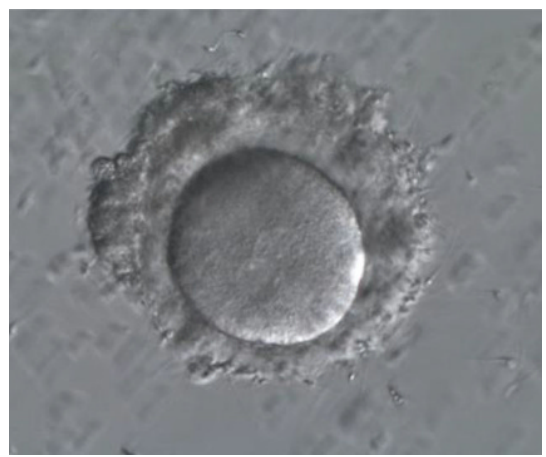

Abb. 4 \& In-vitro-Fertilisation (IVF). (Mit freundlicherGenehmigung von Dr.Min Xie, universitäres Kinderwunschzentrum USZ. Diese Abbildung fällt nicht unter die Creative Commons CC BYLizenz dieser Publikation.)

gilt heutzutage als Standardvorgehen bei männlicher Subfertilität und bei Fertilisationsversagen einer IVF, wird jedoch auch bei einer unerklärten Sterilität angewendet.

Die erste Schwangerschaft mit ICSI konnte im Jahr 1992 erzielt werden [20].

Bevor eine ICSI erfolgen kann, müssen die Oozyten von den Kumuluszellen und der Corona radiata befreit, also denudiert werden. Sofern ein Spermium selektioniert wurde, wird dieses vor der intrazytoplasmatischen Injektion immobilisiert, um nicht die intrazellulären Strukturen der Oozyte zu verletzten. Häufig wird dazu das Spermium zunächst in einen Tropfen Polyvinylpyrrolidon gegeben. Durch dessen erhöhte Viskosität wird die Motilität des Spermiums vermindert. Durch ein ruckartiges Ziehen am Schwanzende mittels einer Mikrokapillare wird das Spermium schliesslich komplett immobilisiert und kann in die Oozyte injiziert werden. Für die Injektion wird die Oozyte durch eine Haltepipette fixiert, sodass sich der erste Polkörper bei 6 oder 12 Uhr befindet. Anschliessend wird das Spermium in der Äquatorialebene injiziert, wie in - Abb. 5 dargestellt. Dadurch wird gewährleistet, dass der Spindelapparat nicht verletzt wird.

16-18 h nach der Durchführung einer IVF oder ICSI kann die Fertilisation kontrolliert werden. Die zu erwartende Fertilisationsrate von reifen Oozyten liegt heutzutage bei ca. $60 \%$. Eine normale Fertilisation ist durch das Vorhandensein von 2 Vorkernen im Zentrum des Zytoplasmas der Oozyte sowie 

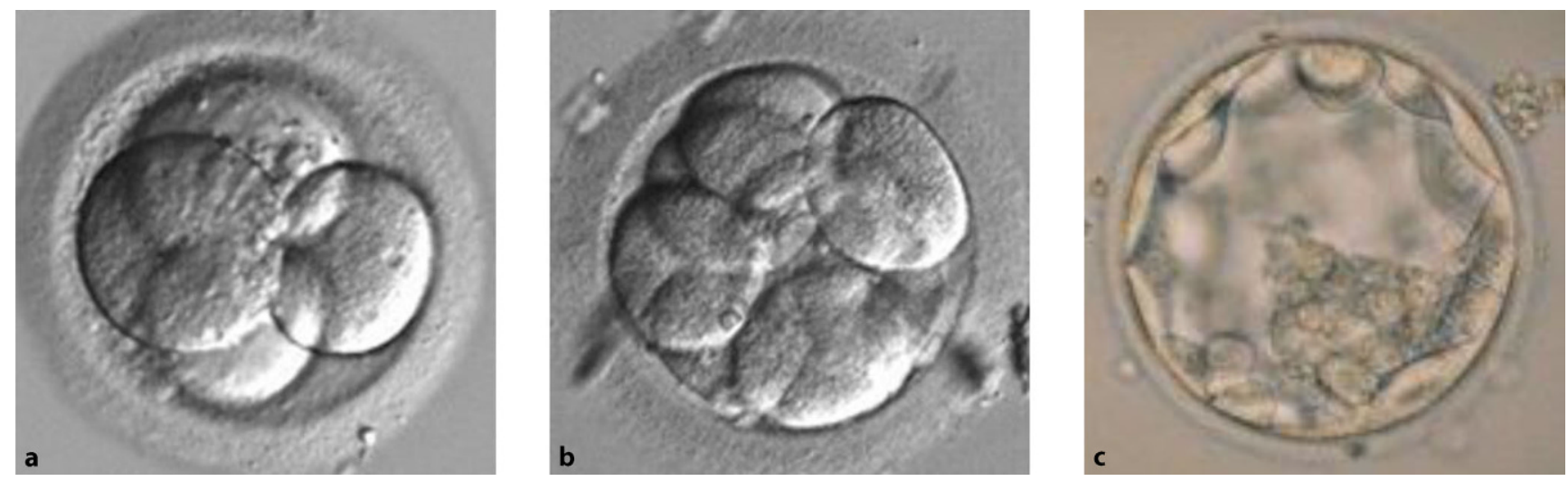

Abb. 7 A Embryonen, a Tag 2, b Tag 3, c Tag 5 (Mit freundlicher Genehmigung von Dr. Min Xie, universitäres Kinderwunschzentrum USZ. Diese Abbildung fällt nicht unter die Creative Commons CC BY-Lizenz dieser Publikation.)

dem Vorliegen von 2 Polkörpern gekennzeichnet. Bei den Vorkernen handelt es sich um das haploide Genmaterial, zum einen aus der Oozyte und zum anderen aus dem Spermium. Diese befruchteten Oozyten werden auch Vorkernstadien genannt (• Abb. 6).

Werden Vorkernstadien weiterentwickelt, entstehen 2 Tage nach der Eizellentnahme optimalerweise ein 4-ZellEmbryo, 3 Tage danach ein 8-Zell-Embryo und 5 Tage danach eine Blastozyste (• Abb. 7).

\section{Lutealphasensupport und Embryonentransfer}

Vor einem Embryonentransfer ist es wichtig, einen Lutealphasensupport $\mathrm{zu}$ beginnen, da die körpereigenen Progesteronlevel bei einer IVF-/ICSI-Behandlung $\mathrm{zu}$ tief sind und daraus schlechte Implantations- und Schwangerschaftsraten resultieren. Verschiedene Medikationen werden bis anhin dafür verwendet: vaginal oder subkutan appliziertes Progesteron, hCG-Injektionen, Progesteron und Östrogenapplikationen sowie die zusätzliche Gabe eines GnRH-Agonisten.

Prinzipiell kann 2, 3 oder 5 Tage nach der Eizellentnahme der Embryonentransfer durchgeführt werden. Hierfür werden bis maximal 12 Vorkernstadien weiterentwickelt und möglichst ein Embryo mit dem besten morphologischen Grading intrauterin transferiert. Die genaue Zahl von 12 hat keinen reproduktionsmedizinischen Grund, sondern stellt eine Vorgabe des schweizerischen Fortpflanzungsmedizingesetztes dar.

Wie viel Tage nach der Follikelpunktion der Transfer erfolgt, sollte individuell festgelegt werden. In einem CochraneReview zeigten sich zwar höhere Lebendgeburtenraten pro Transfer bei einer Blastozyste [21], zu bedenken ist aber, dass nur knapp $50 \%$ aller Vorkernstadien das Blastozystenstadium erreichen [22]. In der Arbeit von Braga et al. war diese Wahrscheinlichkeit bei „low quality embryos" im Weiteren um bis zu $40 \%$ weiter reduziert [23].

Für den Embryonentransfer stehen verschiedene Kathetersysteme kommerziell zur Verfügung. Der Transfer kann unter Ultraschallsicht oder nach vorgängigem Testtransfer erfolgen, um die optimale Platzierung des Embryos, ca. $1-2 \mathrm{~cm}$ unterhalb des Fundus, zu ermöglichen. Unmittelbar nach dem Embryonentransfer kann die Patientin nach Hause gehen.

\section{Kryokonservierung von Embryonen oder Vorkernstadien}

Nicht benötigte Embryonen oder Vorkernstadien können kryokonserviert und in einem sog. Auftauzyklus zu einem späteren Zeitpunkt transferiert werden. Damit hat das Paar die Möglichkeit, ohne vorgängige hormonelle Stimulation und operative Entnahme der Oozyten eine erneute Schwangerschaftschance $\mathrm{zu}$ haben. Es nehmen somit die kumulativen Schwangerschaftschancen zu. Zudem kann mit diesem Vorgehen das Mehrlingsrisiko reduziert werden.
Für die Kryokonservierung stehen heutzutage 2 Verfahren zur Verfügung. Zum einen das „slow freezing“. Hierbei werden die Zellen in Kombination mit einem niedrig konzentrierten Kryoprotektans langsam heruntergekühlt. Die Zeitdauer für diesen Prozess umfasst mehr als 2 h. Das 2. Verfahren stellt die Vitrifikation dar. Hierbei werden die Zellen sehr rasch gefroren. Dieses wird durchgeführt, indem das Vorkernstadium oder der Embryo in eine hohe Konzentration von Kryoprotektans gegeben und nach Verschluss der Paillette in flüssigem Stickstoff extrem schnell heruntergekühlt wird. Hierbei kommt es zum Gefrieren in einem glasähnlichen Zustand. Von Vorteil ist, dass die beim „slow freezing" möglicherweise entstehende Eiskristallisation nahezu vollständig vermieden werden kann. Ferner benötigt die Vitrifikation nur sehr wenig Zeit und keine zusätzlichen Maschinen.

\section{Präimplantationsdiagnostik}

Mit dem revidierten Fortpflanzungsmedizingesetz ist es seit September 2017 erlaubt, eine Präimplantationsdiagnostik am Embryo durchzuführen. So können diese auf monogenetische Defekte (PGT$\mathrm{M})$, strukturelle Chromosomenanomalien (PGT-SR) und Aneuploidien (PGTA) untersucht werden.

Hierzu erfolgt an den Blastozysten eine Trophektodermbiopsie. Nach Eröffnung der Zona pellucida werden ca. 5-10 Trophektodermzellen entnommen (siehe - Abb. 8) 


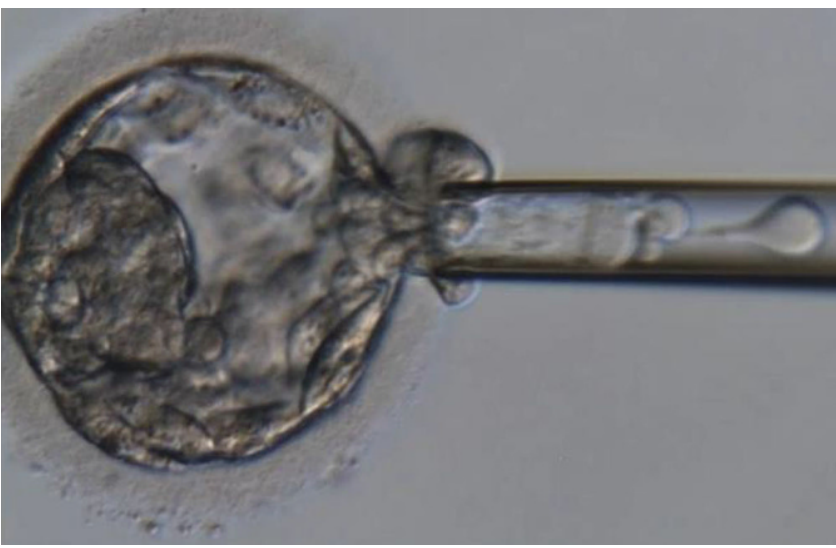

Abb. $8<$ Trophektodermbiopsie (Mit freundlicher Genehmigung von Dr. Min Xie, universitäres Kinderwunschzentrum USZ. Diese Abbildung fällt nicht unter die Creative Commons CC BYLizenz dieser Publikation.)

\begin{tabular}{lll}
\hline Tab. 1 & Symptome, Therapie des OHSS $[27,28]$ & Therapie \\
\hline $\begin{array}{l}\text { Grad I } \\
\text { (mild) }\end{array}$ & $\begin{array}{l}\text { Vergmpö̈serung der Ovarien }<7 \mathrm{~cm} \\
\text { Unwohlsein, Völlegefühl }\end{array}$ & $\begin{array}{l}\text { Regelmässige Ultraschall- } \\
\text { kontrollen, }>2 \text { I Flüssig- } \\
\text { keitszufuhr, eiweiss- und } \\
\text { salzreiche Kost }\end{array}$ \\
$\begin{array}{l}\text { Grad II } \\
\text { (mode- } \\
\text { rat) }\end{array}$ & $\begin{array}{l}\text { Vergrösserung der Ovarien }<12 \mathrm{~cm} \\
\text { Unwohlsein, Völlegefühl, evtl. Diarrhö, Emesis, Aszites }\end{array}$ & $\begin{array}{l}\text { Wie Grad I, zusätzlich } \\
\text { Hämatokrit- und Elek- } \\
\text { trolytkontrollen, tägliche } \\
\text { Gewichtskontrolle }\end{array}$ \\
\hline $\begin{array}{l}\text { Grad III } \\
\text { (schwer) }\end{array}$ & $\begin{array}{l}\text { Ovarien }>12 \mathrm{~cm} \\
\text { Emesis, Erbrechen, evtl. Diarrhö, Aszites und u. U. Hydro- } \\
\text { thorax mit Dyspnoe, Oligurie, Hypovolämie und Hämo- } \\
\text { konzentration, Risiko von thrombembolischen Ereignis- } \\
\text { sen, Elektrolytverschiebung bis hin zur Entgleisung }\end{array}$ & $\begin{array}{l}\text { Stationäre Aufnahme, } \\
\text { symptomatische Thera- } \\
\text { pie }\end{array}$ \\
\hline & $\begin{array}{l} \\
\end{array}$ &
\end{tabular}

Die biopsierten Blastozysten werden nach der Biopsie kryokonserviert, da die genetische Analyse des gewonnenen Materials ein paar Wochen in Anspruch nehmen kann. Nach Erhalt des genetischen Resultates kann anschliessend ein Embryonentransfer im Auftauzyklus erfolgen.

Bei der Aufklärung zu einer Präimplantationsdiagnostik ist die Tatsache zu beachten, dass Blastozysten in 5-15\% der Fälle Mosaike haben können, d.h. unauffällige und pathologische Zelllinien [24]. Somit kann mit keiner vollständigen Sicherheit angenommen werden, dass keine Pathologie beim Kind besteht, wenn die Präimplantationsdiagnostik unauffällig war. Aus diesem Grund besteht die Empfehlung, dennoch eine Pränataldiagnostik durchzuführen.

\section{Komplikationen der IVF-/ICSI- Behandlungen}

In ca. $8 \%$ der Fälle kann es zu einer meist nur schwachen Blutung aus der vagina-
Hämokonzentration, mit dem Risiko von thrombembolischen Ereignissen und reduzierter Endorgandurchblutung, führen [26]. Eingeteilt wird das OHSS in 3 Schweregrade: in eine milde (Grad I), eine moderate (Grad II) und eine schwere Form (Grad III; - Tab. 1).

Die Inzidenz wird in der Literatur sehr unterschiedlich angegeben. Gründe sind zum einen unterschiedliche zugrunde liegende Definitionen und zum anderen unterschiedliche Kollektive [29]. Bezogen auf eine IVF-/ICSI-Behandlung werden für ein mildes OHSS eine Inzidenz von $20-33 \%$, für ein moderates OHSS von $3-6 \%$ und für ein schweres OHSS von $0,5-5 \%$ angegeben [30]. Risikofaktoren für die Entstehung eines OHSS sind ein junges Alter der Patientin, ein niedriger BMI, polyzystische Ovarien, die Anamnese eines früheren OHSS sowie in der Stimulation hohe Östradiolwerte, die Entwicklung zahlreicher Follikel und die Gewinnung von vielen Oozyten [26]. Zur Risikoverminderung kann Metformin, insbesondere bei Frauen mit polyzystischen Ovarien, eingesetzt werden. Eine weitere Möglichkeit der Risikominimierung besteht beim Antagonistenprotokoll. Hier kann die Triggerung der letzten Endausreifung der Oozyten mittels Gabe eines GnRH-Agonisten anstelle von hCG vorgenommen werden. Dies senkt signifikant die Inzidenz für das Auftreten eines OHSS [30]. Allerdings sind die Schwangerschaftsraten bei einem unmittelbar erfolgenden Embryonentransfer deutlich reduziert, weswegen alle Embryonen kryokonserviert werden sollten. Zu einem späteren Zeitpunkt kann dann ein Embryonentransfer im Auftauzyklus ohne Beeinträchtigung der Erfolgschancen erfolgen [31].

Um den zeitlichen Rahmen eines OHSS zu verkürzen, können ferner alle Embryonen nach der Eizellentnahme kryokonserviert werden. Das Auftreten eines OHSS kann damit zwar nicht verhindert werden, aber ca. 14 Tage nach Applikation des hCG zur Triggerung der letzten Eizellreifung kommt es zu einer Abbruchblutung und damit zum raschen Sistieren des OHSS. 


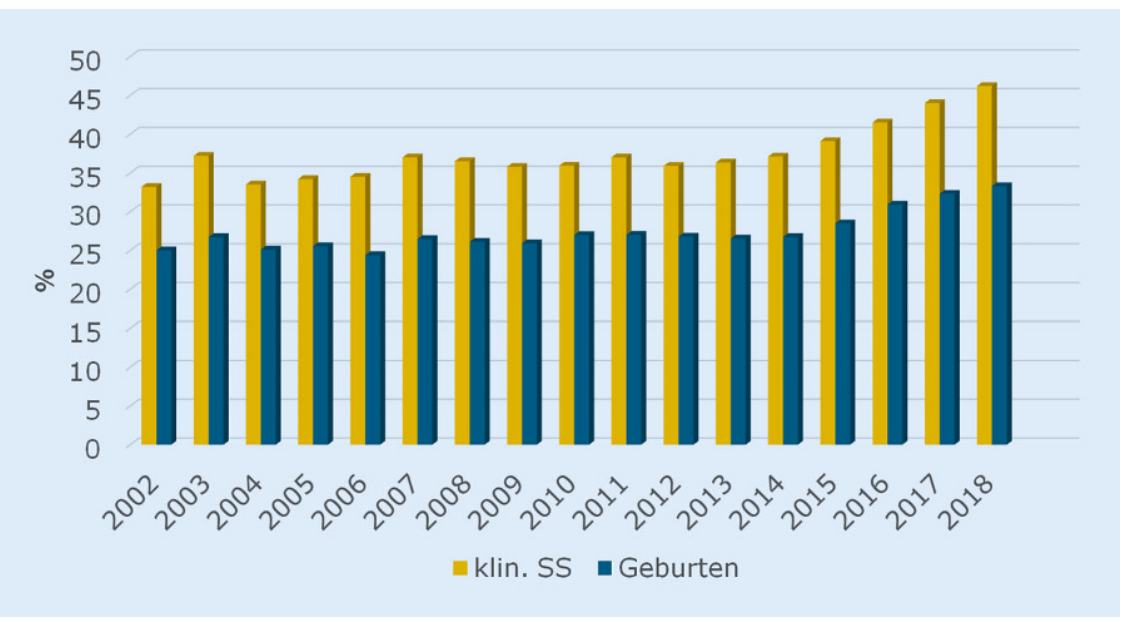

Abb. $9 \Delta$ Schwangerschaftsraten und Lebendgeburtsraten durch IVF/ICSI in der Schweiz pro behandelter Patientin und Jahr. klin. klinisch, ICS/ intrazytoplasmatische Spermieninjektionsbehandlung, IVF In-vitro-Fertilisationsbehandlung, SS Schwangerschaft. (Nach [12])

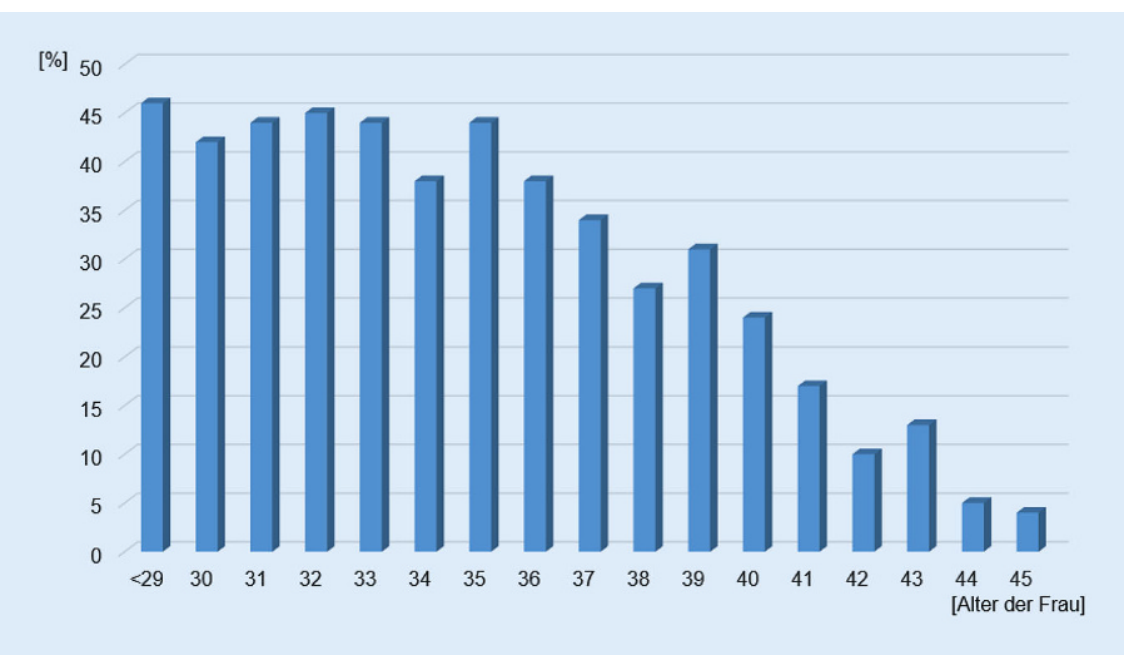

Abb. 10 ॥ Kumulative Lebendgeburtenraten pro Patientin durch IVF/ICSI in der $\mathrm{CH}$ je nach maternem Alter. CH Schweiz, ICS/ intrazytoplasmatische Spermieninjektionsbehandlung, IVF In-vitro-Fertilisationsbehandlung. (Daten wurden von FIVNAT-CH zur Verfügung gestellt)

\section{Erfolgschancen}

Gemäss der aktuellen Veröffentlichung der Daten des Bundesamts für Statistik (BfS 2021) wurden 2018 11.241 Zyklen bei 6012 Paaren durchgeführt, wobei in fast $82 \%$ eine ICSI-Behandlung erfolgte [12].

Die Schwangerschafts- und Lebendgeburtsraten steigen stetig an und lagen in der Schweiz 2018 bei 46,2\% respektive $33,3 \%$ pro behandelter Patientin und Jahr (• Abb. 9).

Werden die Erfolgschancen nach Alter der Frau betrachtet, zeigt sich mit zunehmendem maternen Alter eine sig-
2018 bei 32,7 \% Das universitäre Kinderwunschzentrum des Universitätsspitals Zürich ist eines der ersten Zentren der Schweiz, welches die Zulassung für die Durchführung der PID erhielt. Nach einer Einführungsphase erfolgte im Jahr 2019 bei 19 Paaren die PID mit einer Lebendgeburtenrate von $47 \%$. 2020 wurde bei 28 Paaren die PID mit einer Schwangerschaftsrate von $67 \%$ durchgeführt; eine Lebendgeburtenrate kann nicht angegeben werden, da noch nicht alle Frauen entbunden haben.

\section{Einfluss von IVF-/ICSI- Behandlungen auf das kindliche Outcome}

Bevor eine Sterilitätstherapie durchgeführt wird, ist die Aufklärung des Paares nicht nur über die Chancen der Therapie, sondern auch über die sowohl maternen als auch, bei Eintritt einer Schwangerschaft, kindlichen Risiken von zentraler Bedeutung. Auch über 40 Jahre nach der ersten Geburt nach IVF-Behandlung bleibt die Frage nach den kindlichen Risiken eine der kritischsten Diskussionspunkte.

\section{Mehrlinge}

Erfreulicherweise streben immer mehr Länder eine Reduktion der Mehrlingsraten an, indem vermehrt nur noch ein Embryo transferiert wird. Durch das 2017 in Kraft gesetzte revidierte Fortpflanzungsmedizingesetz der Schweiz, durch das nun nicht mehr alle erzeugten Embryonen im Frischzyklus transferiert werden müssen, zeigt sich eine signifikant reduzierte Mehrlingsrate seit eben diesem Jahr 2017. Waren es in den Jahren von 2006-2016 noch zwischen $15 \%$ und $19 \%$, so sanken die Mehrlingsraten auf 7,8\% im Jahr 2018 und 5,2\% im Jahr 2019 (- Abb. 12).

\section{Perinatale Risiken}

Die sinkenden Mehrlingsraten wirken sich auch auf die perinatalen Risiken positiv aus. Dennoch bestehen leicht erhöhte Risiken gegenüber spontan gezeugten Kindern. Die Aussagen der Studien sind jedoch nicht immer kon- 


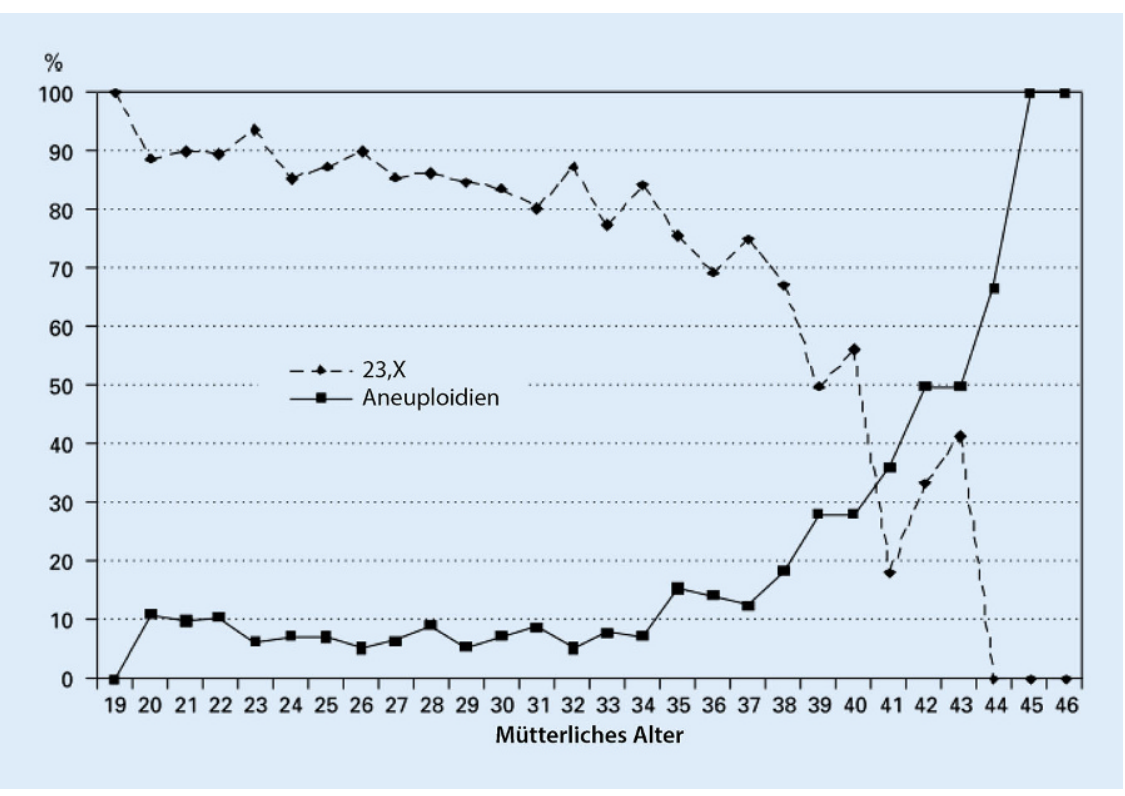

Abb. 11 A Aneuploidierate je nach maternem Alter. (Aus [32], mit Genehmigung von S. Karger AG, Basel. Diese Abbildung fällt nicht unter die Creative Commons CC BY-Lizenz dieser Publikation.)

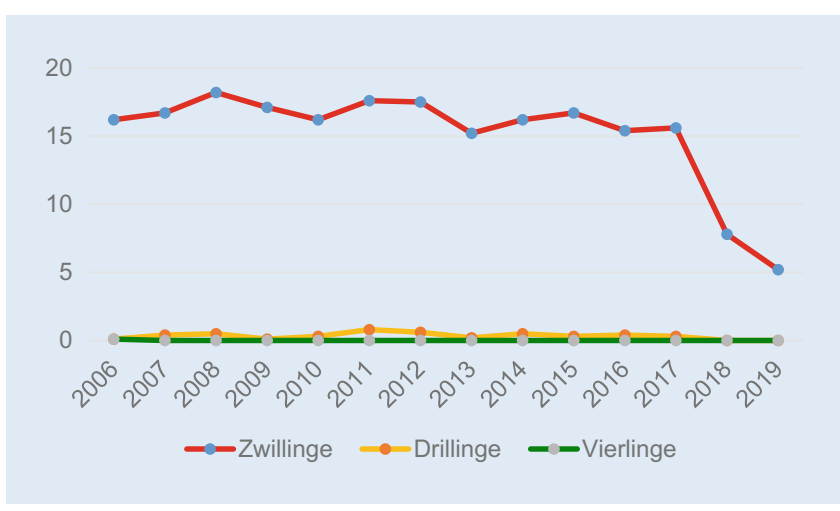

Abb. $12 \triangleleft$ Anzahl der Mehrlingsgeburten in der Schweiz $(\mathrm{CH})$, auf den Daten von FIVNAT-CH basierend. (Mod. nach [33])

gruent, da das Finden einer optimalen Vergleichsgruppe eine grosse Herausforderung darstellt. In einer grossen Reviewarbeit aus Dänemark wurden viele Aspekte der perinatalen Risiken beleuchtet [34].

Viele Studien zeigen ein erhöhtes Risiko für eine Frühgeburt (relatives Risiko [RR] von $1,41-2,04)$ sowie einer extremen Frühgeburt (RR: 1,68-3,07). Ebenso sind die Raten für ein niedriges und sehr niedriges Geburtsgewicht erhöht (RR: 1,6-1,7 und 1,8-3,0). Das relative Risiko für eine perinatale Mortalität liegt zwischen 1,7 und 2,0. Bei der Untersuchung des Outcomes von IVF und ICSI zeigt sich bei ICSI gegenüber IVF ein geringeres Risiko für eine Frühgeburtlichkeit. Eine mögliche Erklärung hierfür ist, dass bei einer ICSI-Behand-
Outcome betreffend Frühgeburt für die Zeiträume 1988-1992, 1993-1997 und 1998-2002 untersucht. Es konnte aufgezeigt werden, dass es zu einem deutlichen Absinken der Risiken von Frühgeburt und extremer Frühgeburt kam, je jünger der untersuchte Zeitraum war [36]. Als Gründe hierfür werden über die Jahre hin veränderte Patientencharakteristika, die Abnahme von Mehrlingen ebenso wie die immer besseren klinischen und labortechnischen Fähigkeiten diskutiert. Auch hat der Zugang zu ART-Behandlungen zugenommen, sodass Paare mit einer kürzeren Dauer einer Sterilität die Therapie erhalten.

Bei der Durchführung einer ICSI-Behandlung zeigen sich leicht erhöhte Raten von chromosomalen Anomalien im Vergleich zu spontan gezeugten Kindern ( 1,6 vs. $0,5 \%)$. Dieses zeigte sich auf für Kinder von Vätern mit einer Oligozoospermie (2,1\% vs. $0,24 \%$; [37]).

Es ist sehr schwierig, eine klare Einschätzung von eventuellen Risiken zu finden, da viele Faktoren eine Rolle spielen. So gibt es Hinweise, dass die Sterilität an sich einen Risikofaktor darstellt. In der Arbeit von Davies et al. zeigte sich für das Auftreten von Malformationen ein erhöhtes relatives Risiko, wenn eine spontane Schwangerschaft eintrat, nachdem vorgängig eine Schwangerschaft mittels IVF-/ICSI-Behandlung oder eine Schwangerschaft bei einer belasteten Sterilitätsanamnese, jedoch ohne Sterilitätstherapie eintrat (RR: 1,25 sowie 1,29; [38]).

Hinsichtlich langfristiger Gesundheitsrisiken ist die aktuell vorliegende Evidenz begrenzt, es gibt aber einzelne Studien, die auf ein erhöhtes Risiko für kardiovaskuläre Erkrankungen hinweisen [34].

Es muss also davon ausgegangen werden, dass ein geringfügig erhöhtes Risiko für Kinder, gezeugt durch IVF/ICSI, besteht. Wichtig ist es, die Paare hierüber aufzuklären. Ferner ist es für weitere Evaluationen von möglichen Langzeitrisiken sinnvoll, die Überwachung von ART-Kindern durch nationale Register zu ermöglichen. 


\section{Fazit für die Praxis}

- Die assistierte Fertilisation verhilft vielen Paaren zur Erfüllung des Kinderwunsches. Die Entwicklung zeigt steigende Erfolgsraten. Das maternale Alter spielt aber nach wie vor eine grosse Rolle. Je älter die Frau wird, umso geringer sind aufgrund einer schlechteren Qualität und zunehmenden Aneuploidierate der Oozyten die Schwangerschaftsraten. Dieses kann auch durch eine IVF/ICSIBehandlung nicht geändert werden. Sinnvoll wäre es, den Verlauf der Fertilität bereits jungen Frauen bewusst zu machen.

- Durch die revidierte Version des Fortpflanzungsmedizingesetztes ist es nun auch in der Schweiz möglich, Verfahren, die früher nur im Ausland erfolgen konnten, im Inland erfolgreich durchzuführen, wie beispielsweise die Präimplantationsdiagnostik. Weiterhin besteht das Bestreben, die ovarielle Stimulation zu optimieren und die Risiken zu senken. Ferner bedarf es fortwährender Analysen der kindlichen Outcomes mit einem Langzeitfollow-up.

\section{Korrespondenzadresse}

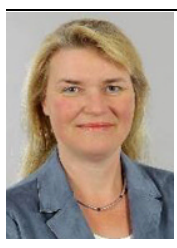

PD Dr. Ruth Stiller

Universitäres Kinderwunschzentrum, Klinik für Reproduktions-Endokrinologie, Universitätsspital Zürich Frauenklinikstrasse 10, 8091 Zürich, Schweiz ruth.stiller@usz.ch

Danksagung. Der Leiterin des IVF Labors des universitären Kinderwunschzentrums des Universitätsspitals Zürich, Frau Dr. Min Xie, danke ich für die zur Verfügungsstellung der Bilder.

Funding. Open access funding provided by University of Zurich

\section{Einhaltung ethischer Richtlinien}

Interessenkonflikt. R. Stiller gibt an, dass kein Interessenkonflikt besteht.

Für diesen Beitrag wurden von der Autorin keine Studien an Menschen oder Tieren durchgeführt.
Für die aufgeführten Studien gelten die jeweils dort angegebenen ethischen Richtlinien.

Open Access. Dieser Artikel wird unter der Creative Commons Namensnennung 4.0 International Lizenz veröffentlicht, welche die Nutzung, Vervielfältigung Bearbeitung, Verbreitung und Wiedergabe in jeglichem Medium und Format erlaubt, sofern Sie den/die ursprünglichen Autor(en) und die Quelle ordnungsgemäß nennen, einen Link zur Creative Commons Lizenz beifügen und angeben, ob Änderungen vorgenommen wurden.

Die in diesem Artikel enthaltenen Bilder und sonstiges Drittmaterial unterliegen ebenfalls der genannten Creative Commons Lizenz, sofern sich aus der Abbildungslegende nichts anderes ergibt. Sofern das betreffende Material nicht unter der genannten Creative Commons Lizenz steht und die betreffende Handlung nicht nach gesetzlichen Vorschriften erlaubt ist, ist für die oben aufgeführten Weiterverwendungen des Materials die Einwilligung des jeweiligen Rechteinhabers einzuholen.

Weitere Details zur Lizenz entnehmen Sie bitte der Lizenzinformation auf http://creativecommons.org/ licenses/by/4.0/deed.de.

\section{Literatur}

1. Zegers-Hochschild F et al (2009) International Committee for Monitoring Assisted Reproductive Technology (ICMART) and the World Health Organization (WHO) revised glossary of ART terminology, 2009. Fertil Steril 92(5):1520-1524

2. Vander Borght M, Wyns C (2018) Fertility and infertility: Definition and epidemiology. Clin Biochem 62:2-10

3. Fritz M, Speroff L (2011) Clinical Gynecologic Endocrinology and Infertility. 8. Aufl., S 1336. ISBN 978-0-7817-7968-5

4. Steiner AZ (2013) Biomarkers of ovarian reserve as predictors of reproductive potential. Semin Reprod Med 31(6):437-442

5. Jain T, Soules MR, Collins JA (2004) Comparison of basal follicle-stimulating hormone versus the clomiphene citrate challenge test for ovarian reserve screening. Fertil Steril 82(1):180-185

6. van der Steeg JW et al (2007) Predictive value and clinical impact of Basal follicle-stimulating hormone in subfertile, ovulatory women. J Clin Endocrinol Metab 92(6):2163-2168

7. Iliodromiti $S$ et al (2014) The predictive accuracy of anti-Mullerian hormone for live birth after assisted conception: a systematic review and meta-analysis of the literature. Hum Reprod Update 20(4):560-570

8. Tal R et al (2015) Antimullerian hormone as predictor of implantation and clinical pregnancy after assisted conception: a systematic review and meta-analysis. Fertil Steril 103(1):119-130.e3

9. Crawford NM, Steiner AZ (2015) Age-related infertility. Obstet Gynecol Clin North Am 42(1):15-25

10. Broekmans FJ et al (2006) A systematic review of tests predicting ovarian reserve and IVF outcome. Hum Reprod Update 12(6):685-718

11. Steptoe PC, Edwards RG (1978) Birth after the reimplantation of a human embryo. Lancet 2(8085):366

12. https://www.bfs.admin.ch/bfs/de/home/ statistiken/gesundheit/gesundheitszustand/ reproduktive/medizinisch-unterstuetzte-

fortpflanzung.html

13. De Geyter C et al (2015) Twenty years' experience with the Swiss data registry for assisted reproductive medicine: outcomes, key trends and recommendations for improved practice. Swiss MedWkly w14087:145

14. Kasius A et al (2014) Endometrial thickness and pregnancy rates after IVF: a systematic review and meta-analysis. Hum Reprod Update 20(4):530-541

15. Zhao J et al (2014) Endometrial pattern, thickness and growth in predicting pregnancy outcome following 3319 IVF cycle. Reprod Biomed Online 29(3):291-298

16. Lenz S, Lauritsen JG, Kjellow M (1981) Collection of human oocytes for in vitro fertilisation by ultrasonically guided follicular puncture. Lancet 1(8230):1163-1164

17. Wikland M, Enk L, Hamberger L (1985) Transvesical and transvaginal approaches for the aspiration of follicles by use of ultrasound. Ann N Y Acad Sci 442:182-194

18. Catt J et al (1994) Subzonal insertion of multiple sperm is a treatment for malefactor infertility. Fertil Steril 61(1):118-124

19. Fishel $S$ et al (1992) Evaluation of 225 patients undergoing subzonal insemination for the procurement of fertilization in vitro. Fertil Steril 57(4):840-849

20. Palermo G et al (1992) Pregnancies after intracytoplasmic injection of single spermatozoon into an oocyte. Lancet 340(8810):17-18

21. Glujovsky D et al (2016) Cleavage stage versus blastocyst stage embryo transfer in assisted reproductive technology. Cochrane Database Syst Rev Cd002118:6

22. Thomas MR et al (2010) Clinical predictors of human blastocyst formation and pregnancy after extended embryo culture and transfer. Fertil Steril 94(2):543-548

23. Braga DP et al (2014) The importance of the cleavage stage morphology evaluation for blastocyst transfer in patients with good prognosis. JAssist Reprod Genet 31(8):1105-1110

24. Zore T et al (2019) Transfer of embryos with segmental mosaicism is associated with a significant reduction in live-birth rate. Fertil Steril 111(1):69-76

25. Bennett SJ et al (1993) Complications of transvaginal ultrasound-directed follicle aspiration: a review of 2670 consecutive procedures. J Assist Reprod Genet 10(1):72-77

26. Smith V, Osianlis T, Vollenhoven B (2015) Prevention of Ovarian Hyperstimulation Syndrome: A Review. Obstet Gynecol Int p:514159

27. Rabau, E., et al., Human menopausal gonadotropins for anovulation and sterility. Results of 7 years of treatment. Am J Obstet Gynecol, 1967. 98(1): p. 92-8.

28. Schenker JG, Weinstein D (1978) Ovarian hyperstimulation syndrome: a current survey. Fertil Steril 30(3):255-268

29. Delvigne A, Rozenberg S (2002) Epidemiology and prevention of ovarian hyperstimulation syndrome (OHSS): a review. Hum Reprod Update 8(6):559-577

30. Mourad S, Brown J, Farquhar C (2017) Interventions for the prevention of OHSS in ART cycles: an overview of Cochrane reviews. Cochrane Database Syst Rev 1(Cd012103): 1

31. Griesinger, G., et al., Ovarian hyperstimulation syndrome prevention by gonadotropin-releasing hormone agonist triggering of final oocyte maturation in a gonadotropin-releasing hormone 


\section{Buchbesprechung}

antagonist protocol in combination with a "freezeall" strategy: a prospective multicentric study. Fertil Steril, 2011.95(6):p. 2029-33, 2033.e1.

32. Pellestor, F., T. Anahory, and S. Hamamah, Effect of maternal age on the frequency of cytogenetic abnormalities in human oocytes. Cytogenet Genome Res, 2005.111(3-4): p. 206-12.

33. De Geyter C (2021) Änderung des Fortpflanzungsmedizingesetzes und daraus resultierende Entwicklungen in der Schweiz. Gynäkologische Endokrinologie. https://doi.org/10.1007/s10304020-00369-0

34. Berntsen $S$ et al (2019) The health of children conceived by ART: 'the chicken or the egg? Hum Reprod Update 25(2):137-158

35. Maheshwari Aetal (2018) Is frozen embryo transfer better for mothers and babies? Can cumulative meta-analysis provide a definitive answer? Hum Reprod Update 24(1):35-58

36. Henningsen AA et al (2015) Trends in perinata health after assisted reproduction: a Nordic study from the CoNARTaS group. Hum Reprod 30(3):710-716

37. Bonduelle $M$ et al (2002) Prenatal testing in ICSI pregnancies: incidence of chromosomal anomalies in 1586 karyotypes and relation to sperm parameters. Hum Reprod 17(10):2600-2614

38. Davies MJ et al (2012) Reproductive technologies and the risk of birth defects. N Engl J Med 366(19):1803-1813

Hinweis des Verlags. Der Verlag bleibt in Hinblick auf geografische Zuordnungen und Gebietsbezeichnungen in veröffentlichten Karten und Institutsadressen neutral.

\section{Markus S. Kupka (Hrsg.)}

\section{Reproduktionsmedizin}

Zahlen und Fakten für die Beratung

Urban \& Fischer Verlag/Elsevier GmbH 2021, 1. Auflage, 136 S., 27 Abb., (ISBN: 9783-437-24944-0), 29,00 EUR

Kaum ein Gebiet der Medizin hat in den letzten Jahrzehnten eine so starke Entwicklung durchgemacht wie die Reproduktionsmedizin. Daher ist es nicht einfach, die sich ständig aktualisierenden Techniken, Fakten und Zahlen im Blick zu behalten, weder für die niedergelassenen Gynäkolginnen/Gynäkologen und Psychosozialen Fachkräfte, die Betroffene mit unerfülltem Kinderwunsch beraten, noch für die Betroffenen selber. Eine Flut von Informationen, mehr oder weniger wissenschaftlich belegt, erreicht die Betroffenen auf unterschiedlichen Kanälen. Eine gute persönliche Beratung zu medizinischen aber auch psychosozialen Faktoren kann die Entscheidungsfindung und Prozesse von Paaren und Personen sehr unterstützen. Falsche Annahmen und Mythen können so korrigiert werden. Das vorliegende Buch, an dem namhafte Medizinerinnen und Mediziner mitgewirkt haben, bietet diese fachlich fundierte Grundlage.

Die aktuelle Datenlage ist sehr übersichtlich mit Hilfe von guten Grafiken und leicht lesbaren Tabellen dargestellt. Damit bietet das kompakte Buch gute Einblicke in viele Themenbereiche der Reproduktionsmedizin. Neben Informationen zur Qualitätssicherung, Datensammlung und zum Verständnis von Statistiken werden interessante Themen wie IVF-Naturelle, Spendersamenbehandlung, Präimplantationsdiagnostik sowie Krebs und Kinderwunsch beleuchtet.
Es wird zudem auf Verteilungsunterschiede der IVF-Zentren in Deutschland eingegangen, wie auf die Optionen und Erfolgschancen in anderen europäischen Ländern. Dem Einfluss des Alters Erstgebärender und der Bedeutung von Schwangerschaften mit 40+ werden eigene Kapitel gewährt. Auch für die Beratung von Frauen mit Adipositas gibt es gute Hinweise (hier wären Beratungsinhalte für anorektische, bulimische oder untergewichtige Patientinnen noch eine gute Ergänzung). Ein eigenes Übersichtskapitel zu den Gründen des unerfüllten Kinderwunsches und eine einleitende Erläuterung aller reproduktionsmedizinischen Techniken wäre (vor allem für nicht-ärztliche Beraterinnen und Berater) noch hilfreich.

Als Reproduktionsmediziner und als Psychologische Psychotherapeutin im Bereich der Gynäkologischen Psychosomatik können wir unseren ärztlichen wie psychosozial beratenden Kolleginnen und Kollegen dieses Buch wärmstens ans Herz legen. Auch für Betroffene, die sich fundiert informieren möchten, ist das Buch durch die meist verständliche Sprache und Erklärungen sehr geeignet.

Prof. Dr. med. Christoph Dorn, Hamburg

Dr. Dipl.-Psych. Almut Dorn, Hamburg 